\title{
Unquenched large orbital magnetic moment in $\mathrm{NiO}$
}

\author{
S. K. Kwon and B. I. Min \\ Department of Physics, Pohang University of Science and Technology, Pohang 790-784, Korea
}

(February 22, 2000)

\begin{abstract}
Magnetic properties of $\mathrm{NiO}$ are investigated by incorporating the spin-orbit interaction in the LSDA $+U$ scheme. It is found that the large part of orbital moment remains unquenched in $\mathrm{NiO}$. The orbital moment contributes about $\mu_{L}=0.29 \mu_{B}$ to the total magnetic moment of $M=1.93 \mu_{B}$, as leads to the orbital-to-spin angular momentum ratio of $L / S=0.36$. The theoretical values are in good agreement with recent magnetic X-ray scattering measurements.
\end{abstract}

PACS number: 71.20.Be, 71.70.Ej, 75.10.Lp, 75.50.Ee

Electronic and magnetic structures of late $3 d$ transition metal (TM) mono-oxides ( $\mathrm{MnO}, \mathrm{FeO}, \mathrm{CoO}$, and $\mathrm{NiO}$ ) have been extensively investigated over the last decades [1]. Conventional band theory with the local spin density approximation (LSDA) fails to describe the electronic structures of the compounds. In the LSDA calculations, the energy gaps of $\mathrm{MnO}$ and $\mathrm{NiO}$ are underestimated [2. Even worse, $\mathrm{FeO}$ and $\mathrm{CoO}$ are predicted to be metallic which are, on the contrary, large gap insulators in nature. The main problem in the LSDA is the use of mean fieldtype exchange-correlation functional which is improper to describe localized $3 d$ electrons.

There have been several theoretical efforts to cure the deficiencies in the LSDA, for example, the self-interaction correction (SIC) scheme [3 5], the GW approximation (GWA) [6, 7], and the LSDA $+U$ method [8, 9]. The SIC-LSDA is in the line of the extended LSDA by removing unphysical electron interaction with itself. In the GWA, the quasi-particle energy is obtained through the self-energy calculation to the lowest order in the screened Coulomb interaction $W$. The GWA method applied to $\mathrm{NiO}$ gives a rather good description of the energy gap size [7]. Computational load, however, is very heavy in the GWA. The LSDA $+U$ method overcomes the failure of the LSDA by incorporating the on-site Coulomb correlation $U$ of the multiband Hubbard model-like. In this method, localized $3 d$ electrons are treated separately from delocalized $s p$ electrons. As a result, all the $3 d \mathrm{TM}$ mono-oxides in the LSDA $+U$ are obtained as insulators with well developed energy gaps which are comparable to experimental values [8]. In this way, the energy gap problem in $3 d$ TM mono-oxides is considered to be solved by various calculational methods.

Due to the outermost characteristics of TM $3 d$ electrons, atomic $3 d$ orbitals are greatly deformed in solids by the crystal field and/or band hybridization effects. Hence, the orbital moment of $3 d \mathrm{TM}$ ion is usually quenched in solids, because it originates from atomic nature of involved atomic elements [10]. For example, all the $3 d$ ferromagnetic transition metals of $\mathrm{Fe}, \mathrm{Co}$, and $\mathrm{Ni}$ show negligible orbital magnetic moments in the range of $\mu_{L} \lesssim 0.1 \mu_{B}$ [1]. It is, however, expected that the strong Coulomb correlation in $3 d$ TM mono-oxides preserves the orbital moment of localized $3 d$ electrons by reducing the ligand crystal field effects at metal ion sites.
In fact, for $\mathrm{CoO}$, it is easily conceived that the orbital moment is only partially quenched because the measured magnetic moment $M=3.4 \mu_{B}$ [12] simply exceeds the spin magnetic moment alone and the minority $t_{2 g}$ band is occupied only two-thirds of its available states. Therefore, the existence of orbital moment in $\mathrm{CoO}$ has been stressed many times 2, 5, 13. It is also shown that the LSDA $+U$ method gives a good description of magnetic structure of $\mathrm{CoO}$ with a large orbital moment of $\mu_{L} \sim 1 \mu_{B}$ [14]. On the other hand, in the case of $\mathrm{NiO}$, measured magnetic moments are in the range of $M=1.77 \sim 2.2 \pm 0.2 \mu_{B}$ 15 17, which are comparable to the spin only moment of isolated $\mathrm{Ni}^{2+}$ ion. Therefore, the magnetic moment in $\mathrm{NiO}$ has been fully attributed to the spin moment and the orbital moment is expected to be completely quenched.

However, recent magnetic X-ray scattering measurement [17] indicates that the orbital-to-spin angular momentum ratio in $\mathrm{NiO}$ is as large as $L / S=0.34$, far from fully quenched orbital moment. In the nonresonant magnetic X-scattering, the separation of spin and orbital moment is possible, because the spin and orbital moment densities have different geometrical prefactors in the scattering cross section that can be adjusted by changing either the scattering geometry or the X-ray polarization. In Ref. [17], the orbital moment in $\mathrm{NiO}$ is extracted by the polarization analysis of nonresonant magnetic-scattering intensities. This method has evidenced a large contribution of the orbital moment to the total magnetic moment. They also found that the spin and orbital moments in $\mathrm{NiO}$ are collinear.

Magnetic moment is one of the basic ground state quantities which should be provided by an appropriate band method. If the orbital moment is so large in $\mathrm{NiO}$, all the previous attempts [8, 18, 19] which tried to directly compare calculated spin moments with experimental moments are under a mistake. The linearized muffin-tin orbital (LMTO) calculation for $\mathrm{NiO}$ with an orbital-polarization (OP) correction (LSDA + OP) in a crystal-field basis yields the spin and orbital magnetic moments of $\mu_{S}=1.43 \mu_{B}$ and $\mu_{L}=0.12 \mu_{B}$, respectively [20]. Although the total magnetic moment is significantly improved to $M=1.55 \mu_{B}$ with the OP correction, as compared to $M=1.23 \mu_{B}$ from the LSDA, it is still smaller than the experimental measurements and 


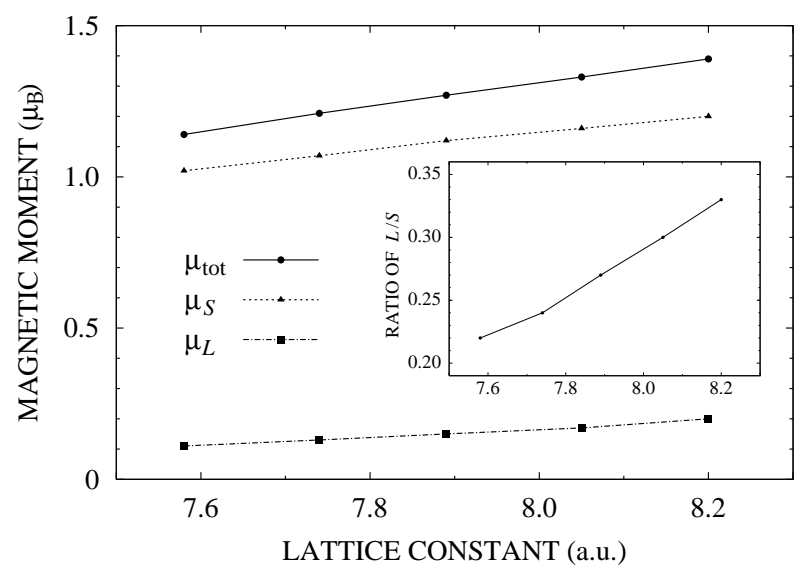

FIG. 1. Magnetic moment behavior with varying the lattice constant in the LSDA. Inset is the behavior of the orbital-to-spin angular momentum ratio $L / S$. Increase of all the magnetic moments is understood by the reduction of the crystal field and the band hybridization strengths with volume expansion.

the ratio of $L / S=0.17$ is only half of the magnetic X-ray scattering value [17]. The SIC-LSDA method [5] yields a large orbital moment of $\mu_{L}=0.27 \mu_{B}$, which, however, was suspected as a methodological artifact by other authors [8, 18]. In more recent study, the orbital moment is simply neglected 19.

To determine the size of orbital moment in $\mathrm{NiO}$, we have performed the LSDA $+U$ calculations using the LMTO band method within the atomic sphere approximation (ASA). The incorporation of the spin-orbit coupling into the LSDA $+U$ method is known to give right orbital polarization in strongly correlated electron systems [14]. The LSDA $+U$ Hamiltonian is given by

$$
\mathcal{H}_{\mathrm{LSDA}+U}=\mathcal{H}_{\mathrm{LSDA}}-\mathcal{H}_{\mathrm{dc}}+\mathcal{H}_{U}
$$

where the first term in the right hand side is the LSDA Hamiltonian and the second term is the double counting correction for the third term $\mathcal{H}_{U}$. With the Coulomb interaction $U$ and exchange interaction $J$ parameters, one can write $\mathcal{H}_{\mathrm{dc}}$ and $\mathcal{H}_{U}$, respectively, as

$$
\begin{aligned}
\mathcal{H}_{\mathrm{dc}}= & \frac{1}{2} U N(N-1)-\frac{1}{2} J \sum_{\sigma} N^{\sigma}\left(N^{\sigma}-1\right) \\
\mathcal{H}_{U}= & \frac{1}{2} \sum_{\{m\}, \sigma} V\left(m m^{\prime} ; m^{\prime \prime} m^{\prime \prime \prime}\right) n_{m m^{\prime \prime}}^{\sigma} n_{m^{\prime} m^{\prime \prime \prime}}^{-\sigma} \\
+ & \frac{1}{2} \sum_{\{m\}, \sigma}\left[V\left(m m^{\prime} ; m^{\prime \prime} m^{\prime \prime \prime}\right)-V\left(m m^{\prime} ; m^{\prime \prime \prime} m^{\prime \prime}\right)\right] \\
& \times n_{m m^{\prime \prime}}^{\sigma} n_{m^{\prime} m^{\prime \prime \prime}}^{\sigma}
\end{aligned}
$$

where $n_{m m^{\prime}}^{\sigma}$ is the $d$ occupation number matrix of spin $\sigma$ and $N^{\sigma}=\operatorname{Tr}\left(n_{m m^{\prime}}^{\sigma}\right), N=N^{+}+N^{-}$. We relate the screened Coulomb interaction $V\left(m m^{\prime} ; m^{\prime \prime} m^{\prime \prime \prime}\right)$ with the Slater integral $F^{k}$;

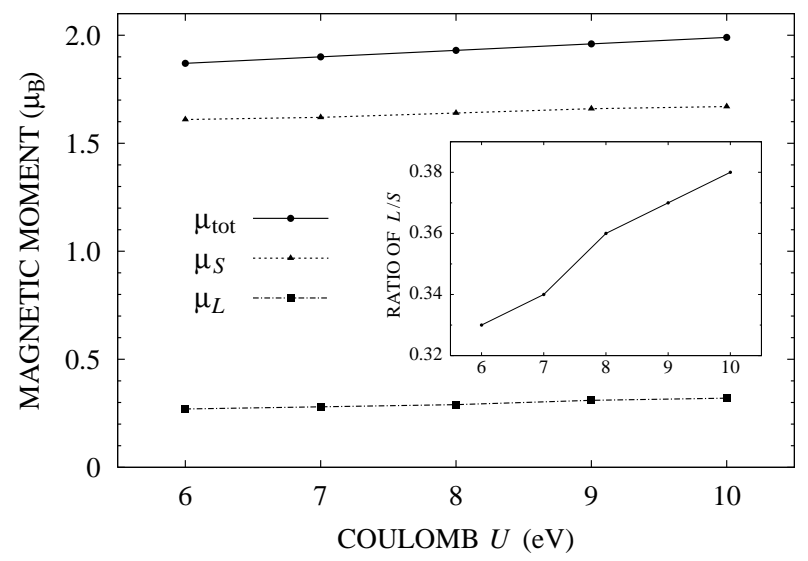

FIG. 2. Coulomb correlation effects on the magnetic moments obtained by the LSDA $+U$ method. We have used the exchange parameter of $J=0.89 \mathrm{eV}$. Inset is the orbital-to-spin angular momentum ratio $L / S$. The total magnetic moment $M=1.93 \mu_{B}$ and the ratio $L / S=0.36$ for $U=8.0 \mathrm{eV}$ are in good agreement with experimental values.

$$
V\left(m m^{\prime} ; m^{\prime \prime} m^{\prime \prime \prime}\right)=\sum_{k=0}^{2 l} c^{k}\left(l m, l m^{\prime \prime}\right) c^{k}\left(l m^{\prime}, l m^{\prime \prime \prime}\right) F^{k},
$$

where $c^{k}\left(l m, l m^{\prime}\right)$ is a Gaunt coefficient. For $3 d$ electrons, three Slater integrals of $F^{0}, F^{2}$, and $F^{4}$ are involved in the calculation. Among those, the ratio of $F^{4} / F^{2}$ is known to be constant $\sim 0.625$ for most $3 d \mathrm{TM}$ atoms 21]. Hence, the actual number of parameters is reduced from three $F^{k}$ 's to two of $U$ and $J$, which are given by $U=F^{0}$ and $J=\left(F^{2}+F^{4}\right) / 14$, respectively. To determine the orbital moment, the spin-orbit coupling is simultaneously included in the self-consistent variational loop 111. We have assumed the antiferromagnetic ordering state of type-II, and the experimental lattice constant of $a=7.893$ a.u. is used.

In Fig. 11, magnetic moment behavior is shown with varying the lattice constant in the LSDA. Both the spin and orbital magnetic moments increase monotonically with increasing the lattice constant. This feature is understandable in view of that the crystal field strength at $\mathrm{Ni}$ sites and the hybridization between $\mathrm{Ni} 3 d$ and $\mathrm{O} 2 p$ bands are reduced with volume expansion. At the experimental lattice constant of $a=7.893$ a.u., the spin and orbital magnetic moments are obtained as $\mu_{S}=1.12 \mu_{B}$ and $\mu_{L}=0.15 \mu_{B}$, respectively, which are consistent with existing results [20]. Both the spin and orbital magnetic moments are larger in $\mathrm{NiO}$ than in fcc Ni which has $\mu_{S}=0.63 \mu_{B}$ and $\mu_{L}=0.06 \mu_{B}$. This suggests that $3 d$ electrons are more localized in $\mathrm{NiO}$ than in $\mathrm{Ni}$ metal. Although the ratio of $L / S=0.27$ in the LSDA is only slightly smaller than $L / S=0.34$ in the experiment (see Table. II), the spin and orbital polarizations in the LSDA are not large enough because of underestimation of the Coulomb correlation between $3 d$ electrons.

Figure 2 shows the Coulomb correlation effects on the 
TABLE I. Calculated spin $\left(\mu_{S}\right)$, orbital $\left(\mu_{L}\right)$, and total $(M)$ magnetic moments in $\mu_{B}$ at the experimental lattice constant of $a=7.893$ a.u.. $L / S$ is the orbital-to-spin angular momentum ratio.

\begin{tabular}{cccccc}
\hline \hline Method & LSDA $^{a}$ & LSDA + OP $^{b}$ & SIC-LSDA $^{c}$ & ${\text { LSDA }+U^{d}}^{\text {Experiment }}$ \\
\hline$\mu_{S}$ & 1.12 & 1.43 & 1.53 & 1.64 & $1.90 \pm 0.2^{e}$ \\
$\mu_{L}$ & 0.15 & 0.12 & 0.27 & 0.29 & $0.32 \pm 0.05^{e}$ \\
$M$ & 1.27 & 1.55 & 1.80 & 1.93 & $1.77^{f}, 1.90^{g}, 2.2 \pm 0.2^{e}$ \\
$L / S$ & 0.27 & 0.17 & 0.35 & 0.36 & $0.34^{e}$ \\
\hline \hline
\end{tabular}

a Present results.

${ }^{b}$ Reference 20].

${ }^{c}$ Reference [5].

${ }^{d}$ Present results with $U=8.0 \mathrm{eV}$ and $J=0.89 \mathrm{eV}$.

$e$ Reference 17 .

$f$ Reference |15.

$g$ Reference 16].

magnetic moments obtained by the LSDA $+U$ method. We have used the exchange parameter of $J=0.89 \mathrm{eV}$ which is comparable to literature value $\mathbb{8}]$. Once the strong Coulomb interaction is introduced between $\mathrm{Ni} 3 d$ electrons, the magnetic moments increase substantially. The role of the Coulomb interaction is significant to localize Ni $3 d$ electrons. With $U=8.0 \mathrm{eV}$ found in Ref. [8] and Ref. [22], we have obtained the spin and orbital magnetic moments of $\mu_{S}=1.64 \mu_{B}$ and $\mu_{L}=0.29 \mu_{B}$, respectively. The total magnetic moment $M=1.93 \mu_{B}$ and the ratio $L / S=0.36$ for $U=8.0 \mathrm{eV}$ are in good agreement with the experimental data. The change of the Coulomb parameter $U$ by $1.0 \mathrm{eV}$ results in increments of both the magnetic moments and the ratio $L / S$ by $\Delta \mu_{S} \sim \Delta \mu_{L} \sim 0.01 \mu_{B}$ and $\Delta(L / S) \sim 0.01$, respectively. The overall total magnetic moment difference and the ratio $L / S$ difference are only $\Delta M=0.12 \mu_{B}$ and $\Delta(L / S)=0.05$ inbetween $U=6.0$ and $10 \mathrm{eV}$. Thus, all the magnetic moments and the ratio $L / S$ are rather insensitive to the Coulomb interaction $U$ within the employed parameter range.

Calculated magnetic moments are summarized in Table. [1] in comparison with experimental data and previous results by other calculational methods. As mentioned above, the sizes of magnetic moments are underestimated in the LSDA. The total magnetic moment of $M=1.27 \mu_{B}$ in the LSDA is much smaller than $M=1.77 \sim 2.2 \pm 0.2 \mu_{B}$ in experiments [15 17]. In the LSDA + OP, the spin moment is significantly improved to $\mu_{S}=1.43 \mu_{B}$ [20], which, however, is still smaller than the experimental values. More serious is the decrease of orbital moment in the LSDA + OP, which unfavorably makes the consistency between the theoretical and experimental $L / S$ ratio become worse. Both the SIC-LSDA $\left(M=1.80 \mu_{B}\right)$ [5] and the LSDA $+U\left(M=1.93 \mu_{B}\right)$ give values in agreement with experimental data. But it can be concluded that the LSDA $+U$ results with $U=8.0$ $\mathrm{eV}$ and $J=0.89 \mathrm{eV}$, among various calculations, are especially in best agreement with experimental measurements. The ratio of $L / S=0.36$ in the LSDA $+U$ is also consistent with $L / S=0.34$ in the magnetic X-ray scattering measurement 17].

In conclusion, we have found using the LSDA $+U$ calculations that the orbital moment in $\mathrm{NiO}$ is not fully quenched, which is as large as $\mu_{L}=0.29 \mu_{B}$. Both the total magnetic moment of $M=1.93 \mu_{B}$ and the orbitalto-spin angular momentum ratio of $L / S=0.36$ for $U=$ $8.0 \mathrm{eV}$ are close to experimental values. The Coulomb correlation and the spin-orbit coupling are crucial to get right magnetic polarization in $\mathrm{NiO}$.

Acknowledgements - The authors would like to thank K.B. Lee for helpful discussions. This work was supported by the KOSEF (1999-2-114-002-5) and in part by the Korean MOST-FORT fund.

[1] L. F. Mattheiss, Phys. Rev. B 5, 290 (1972).

[2] K. Terakura, A. R. Williams, T. Oguchi, and J. Kübler, Phys. Rev. Lett. 52, 1830 (1984); K. Terakura, T. Oguchi, A. R. Williams, and J. Kübler, Phys. Rev. B 30, 4734 (1984).

[3] R. D. Cowan, Phys. Rev. 164, 54 (1967); I. Lindgren, Int. J. Quantum Chem. 5, 411 (1971).

[4] A. Zunger, J. P. Perdew, and G. L. Oliver, Solid State Commun. 34, 933 (1980); J. P. Perdew and A. Zunger, Phys. Rev. B 23, 5048 (1981).

[5] A. Svane and O. Gunnarsson, Phys. Rev. Lett. 65, 1148 (1990).

[6] L. Hedin and S. Lundqvist, in Solid State Physics, edited by H. Ehrenreich, F. Seitz, and D. Turnbell (Academic, New York, 1969), Vol 23, p. 1.

[7] F. Aryasetiawan and O. Gunnarsson, Phys. Rev. Lett. 74, 3221 (1995).

[8] V. I. Anisimov, J. Zaanen, and O. K. Andersen, Phys. Rev. B 44, 943 (1991).

[9] A. I. Liechtenstein, V. I. Anisimov, and J. Zaanen, Phys. Rev. B 52, R5467 (1995). For review, see V. I. Anisimov, F. Aryasetiawan, and A. I. Liechtenstein, J. Phys. : Condens. Matter 9, 767 (1997).

[10] C. Kittel, Introduction to Solid State Physics 7th ed., 
(John Wiley \& Sons, New York, 1996), Chapter 14.

[11] B. I. Min and Y.-R. Jang, J. Phys.: Condens. Matter 3, 5131 (1991).

[12] D. C. Khan and R. A. Ericksson, Phys. Rev. B 1, 2243 (1970).

[13] T. Shishidou and T. Jo, J. Phys. Soc. Jpn. 67, 2637 (1998).

[14] I. V. Solovyev, A. I. Liechtenstein, and K. Terakura, Phys. Rev. Lett. 80, 5758 (1998).

[15] B. E. F. Fender, A. J. Jacobson, and F. A. Wegwood, J. Chem. Phys. 48, 990 (1968).

[16] A. K. Cheetham and D. A. O. Hope, Phys. Rev. B 27, 6964 (1983).

[17] V. Fernandez, C. Vettier, F. de Bergevin, C. Giles, and W. Neubeck, Phys. Rev. B 57, 7870 (1998); W. Neubeck, C. Vettier, V. Fernandez, F. de Bergevin, and C Giles, J. Appl. Phys. 85, 4847 (1999).

[18] M. D. Towler, N. L. Allan, N. M. Harrison, V. R. Saunders, W. C. Mackrodt, and E. Aprà, Phys. Rev. B 50, 5041 (1994).

[19] A. B. Shick, A. I. Liechtenstein, and W. E. Pickett, Phys. Rev. B 60, 10763 (1999).

[20] M. R. Norman, Phys. Rev. B 44, 1364 (1991).

[21] F. M .F. de Groot, J. C. Fuggle, B. T. Thole, and G. A. Sawatzky, Phys. Rev. B 42, 5459 (1990).

[22] M. R. Norman and A. J. Freeman, Phys. Rev. B 33, 8896 (1986). 